\title{
DATA MINING ANALYSIS FOR NATIONAL SECURITY
}

\section{R. E. OKONIGENE ${ }^{1}$, M. J. E. EVBOGHAI ${ }^{2}$ \& GUIAWA MATHURINE ${ }^{3}$}

${ }^{I}$ Professor, Department of Electrical \& Electronics Engineering, Ambrose Alli University, Ekpoma, Edo State, Nigeria

${ }^{2}$ Department of Electrical \& Electronics Engineering, Ambrose Alli University, Ekpoma, Edo State, Nigeria

${ }^{3}$ Department of Electrical/Electronic Engineering, Faculty of Engineering and

Technology Ambrose-Alli University, Ekpoma Edo State, Nigeria

\begin{abstract}
This work takes a view on different data mining and geospatial modeling analysis in the identification process study. The structure and working principle are reviewed alongside recent development in Biometric process. Data mining is a technique that enables registration of citizen from childbirth to the age of 18 upward, while keeping tract with the physical appearance of the child plus his historical background. A database comprises of densely interconnected computer processors working simultaneously (in parallel). A key feature of neural networks is that they are programmed to 'learn' by sifting data repeatedly, looking for relationships to build mathematical models, and automatically correcting these models to refine them continuously.

KEYWORDS: Computer, Processor, Feature, Data, Relationships, Mathematical Models, Automatically \& Models
\end{abstract}

Received: Jan 31, 2017; Accepted: Feb 25, 2017; Published: May 19, 2017; Paper Id.: IJCSEITRJUN20173

\section{INTRODUCTION}

The importance of information for the effective functioning of any enterprise has been a central concern of economic theory for some time. A large body of literature has emerged in an effort to explain how asymmetric and costly information search could explicate equilibrium price dispersion for homogeneous goods [7]. Access to telecommunication for example, has been cited as a factor for social economic development, especially in rural areas (Samuel et al., 2005).

Telecommunications can involve any wired or wireless technologies that allow people and businesses to communicate with each other. Computer networks are only a part of what we learn about; this area of study also covers the security issues and business needs commonly associated with telecommunications. [8]

Mandatory nationwide identification systems have been implemented in a number of countries, including Argentina, Belgium, Colombia, Germany, Italy, Peru, and Spain. While these schemes vary by country, individuals are typically assigned an ID number, which is used for a broad range of identification purposes. Large amounts of personal data such as name, birth date, place of birth, gender, eye color, height, current address, photograph, and other information is linked to this ID number and stored in a centralized database. In many countries, such as Argentina, national ID regimes are adopted during military or authoritarian regimes.

National ID cards and the databases behind them comprise the cornerstone of government surveillance systems that creates risks to privacy and anonymity. The requirement to produce identity cards on demand habituates citizens into participating in their own surveillance and social control. 


\section{METHODS}

\section{Area / Location of the Study}

Edo State Police Command Headquarter is situated on Adesuwa Grammar School Road, Benin City, Edo State, Nigeria. Geographically, the coordinates of Benin City may think of having a latitude of $6^{\circ} 20^{\prime} 17^{\prime \prime}$ N, a Longitude of $5^{\circ} 37^{\prime} 32^{\prime \prime}$ E and an Elevation above sea level of $88 \mathrm{~m}=288 \mathrm{ft}$.

The rate of crime occurrence on a monthly basis was obtained for a period of six years (2010 to 2015) from major crimes and local acts returns for the Edo State Police Command Headquarter “inside State CID”.

The major crime cases reported are namely; Murder, Arm/Robbery, Attempt/Murder, Gravious Harm/Wounding, Assault, Stealing, Rape and indecent Assault, Kidnapping, Cultism, Burglary, House Braking, Store Braking, False Pretence/Cheating, Forgery, Receiving stolen property, Unlawful possession, Arson, Breach of public peace, offences against fire arms act, snatched of vehicles, car theft, vandalization, other offences, S.U.D., offences against traffic act, malicious damage, threatening violence, communal clash, political violence, suicide.

\section{RESULTS}

Table 1: Major Crimes and Local Acts Returns for the Edo State Police Command Head Quarter: 2010(January-December)

\begin{tabular}{|c|c|c|c|c|c|c|c|c|c|c|c|c|c|}
\hline MONTHS & JAN & FEB & MAR & $\mathrm{AP}$ & MAY & JUNE & $\mathrm{JULY}$ & $\mathrm{AU}$ & SE? & $\mathrm{OCT}$ & $\mathrm{NOV}$ & $\mathrm{DEC}$ & TOTAL \\
\hline \multicolumn{14}{|l|}{ OFFENCFS } \\
\hline MURDER & 4 & 5 & 9 & 3 & 8 & 5 & 6 & 5 & 5 & 4 & 5 & 3 & 62 \\
\hline$\triangle R M$ RROBRFRY & 6 & 4 & 7 & 6 & 9 & 4 & $\frac{6}{6}$ & 4 & 6 & 9 & 7 & 7 & 73 \\
\hline ATTEMPTIMURDER & . & 2 & 1 & . & 4 & 2 & . & 1 & 2 & . & . & . & 12 \\
\hline GRAVIOUS HARM/WOUNDING & 14 & 11 & 22 & 12 & 14 & 20 & 13 & 14 & 14 & 29 & 15 & 17 & 195 \\
\hline ASSAULT & 28 & 25 & 31 & 20 & 20 & 25 & 20 & 21 & 18 & 133 & 18 & 24 & 383 \\
\hline \multicolumn{14}{|l|}{ STEALING } \\
\hline RAPFAND INDFCFNTG $\triangle S S A L I I T$ & 4 & 3 & 7 & 6 & 7 & 7 & 4 & 5 & 2 & 8 & 7 & 5 & 64 \\
\hline KIDNAPPING & 3 & 4 & 8 & 5 & 8 & 6 & 4 & 5 & 7 & 1 & 8 & 8 & 67 \\
\hline \multicolumn{14}{|l|}{ CIITISM } \\
\hline BURGLARY & 6 & 5 & 8 & 6 & 7 & 12 & 6 & 7 & 5 & 7 & 3 & 4 & 76 \\
\hline HOUSE BRAKING & 6 & 5 & 6 & 7 & 8 & 8 & $j$ & 4 & 7 & 10 & 6 & 3 & 75 \\
\hline STORF BRFAKING & 5 & 6 & 4 & 4 & 3 & 9 & 3 & 5 & 4 & 6 & 2 & 3 & 54 \\
\hline EALSE PRETENSE/CHEATING & 11 & 8 & 10 & 10 & 14 & 12 & 10 & 12 & 12 & 25 & 10 & 8 & 142 \\
\hline FORGiFRY & - & 1 & 1 & 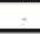 & - & - & 3 & - & - & - & 3 & - & 8 \\
\hline RECEIVING STOLEN PROPERTY & 1 & . & $\therefore$ & $\cdot$ & - & $\therefore$ & . & 3 & 2 & 1 & . & $\therefore$ & 7 \\
\hline UNLAWFUL. PUSSESSION & 4 & - & 10 & - & 2 & - & 2 & 2 & 4 & - & - & 3 & 27 \\
\hline ARSON & . & - & 2 & 2 & . & . & . & 1 & 1 & 2 & . & 2 & 10 \\
\hline EREACH OF PUBLIC PEACE & 6 & 5 & 7 & 8 & 10 & 8 & 8 & 6 & 5 & 32 & 5 & 8 & 108 \\
\hline \multicolumn{14}{|l|}{ OFTEVCES AGAINST FIRE ARMS } \\
\hline \multicolumn{14}{|l|}{ SNATCHED OF VEHICLES } \\
\hline CAR THEFT & 24 & 29 & 20 & 25 & 22 & 28 & 22 & 20 & 16 & 117 & 20 & 27 & 370 \\
\hline \multicolumn{14}{|l|}{ VANLALIZATION } \\
\hline OTHER OFFENCES & 7 & 8 & 14 & 14 & 12 & 14 & $\dot{b}$ & 8 & 8 & 12 & 8 & 7 & 118 \\
\hline \multicolumn{14}{|l|}{ S.U.D } \\
\hline \multicolumn{14}{|l|}{ OFFENCES AGAINST TRAFFIC ACT } \\
\hline MALICIOUS DAMAGE & 1 & 2 & . & $\therefore$ & - & 1 & 2 & $\cdot$ & $\therefore$ & $\dot{.}$ & . & . & 6 \\
\hline \multicolumn{14}{|l|}{ IHREATENING VIOLENCE } \\
\hline COMMUNAL CLASH & - & . & . & . & . & . & . & . & . & . & 1 & - & 1 \\
\hline POLITICAL VIOLENCL & - & - & - & $\cdot$ & - & 1 & 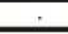 & $\dot{2}$ & $\therefore$ & - & . & - & 1 \\
\hline SIICII)E & - & - & - & 1 & - & 2 & + & 1 & 1 & 2 & - & 2 & 9 \\
\hline
\end{tabular}




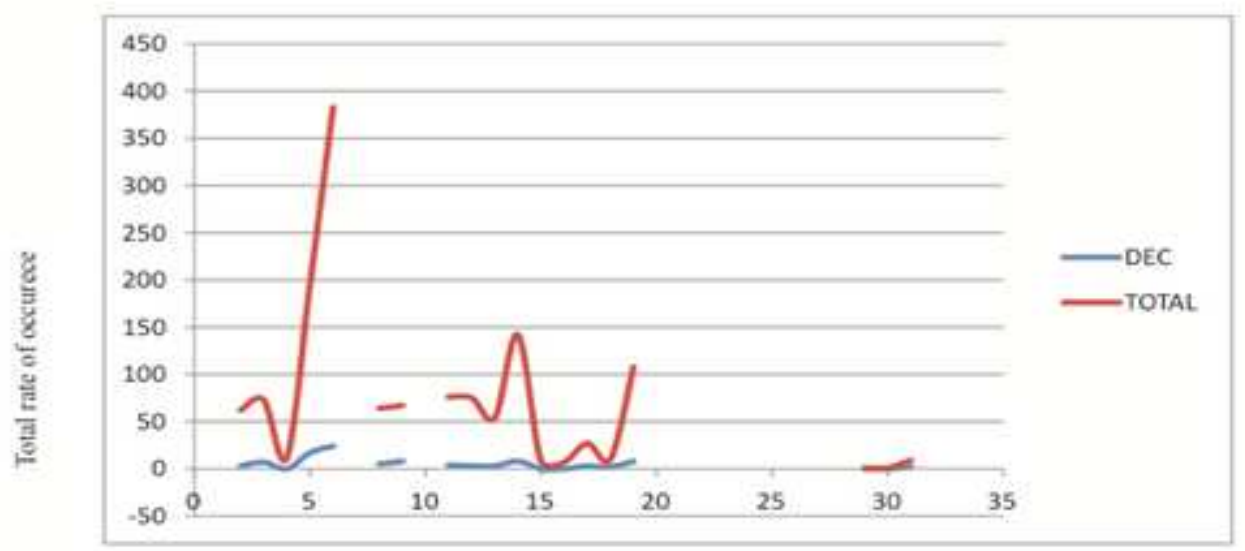

Figure 1(a): Frequency of Occurrence

Table 2: Major Crimes and Local Acts Returns for the Edo State Police Command Headquarter 2010(January-December)

\begin{tabular}{|c|c|c|c|c|c|c|c|c|c|c|c|c|c|}
\hline MONTHS & $\mathrm{J} \Lambda \mathrm{N}$ & FEB & MAR & $\Delta P$ & MAY & JNE & JUL & $\mathrm{AU}$ & SEP & OCT & $\mathrm{NOV}$ & $\mathrm{DEC}$ & TOTAL \\
\hline \multicolumn{14}{|l|}{ RECORDS } \\
\hline $\mathrm{N}^{0}$ OF CASES REPORTED & 130 & 123 & 165 & 129 & 148 & 164 & 120 & 124 & 119 & 398 & 117 & 134 & 1871 \\
\hline \multicolumn{14}{|l|}{ 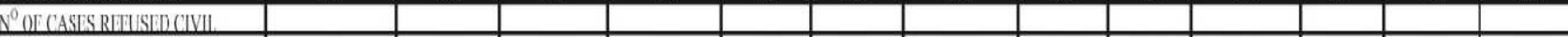 } \\
\hline$\Sigma^{0}$ OE SUSPECTS ARRESTED & 89 & 102 & 112 & 81 & 99 & 97 & 123 & 127 & 117 & 268 & 132 & 136 & 1483 \\
\hline $\mathrm{N}^{0}$ OE PERSONSPROSSECUTE) & 75 & 87 & 98 & 77 & 90 & 89 & 95 & 75 & 74 & 233 & 79 & 85 & 1157 \\
\hline $\mathrm{N}^{0}$ OEPERSONS KULED & 5 & 5 & 11 & 4 & 8 & 5 & 6 & 7 & 5 & 4 & 5 & 5 & 70 \\
\hline \multicolumn{14}{|l|}{ NOAE PERSONSINUIRED } \\
\hline VAIIIE () PROPFRTY ST()LIN ()R & & & & & & & 11452500 & 11979980 & 102685000 & 2892000 & $11(1725590$ & 148114012 & 62476932 \\
\hline VALUE OF PROPERTY & & & & & & & 5878620 & 5457200 & 5135000 & 11221241 & 5009900 & $7,439,500$ & 40141461 \\
\hline NoF CASES UUNDER & 58 & 42 & 72 & 58 & 64 & 77 & 35 & 60 & 48 & 69 & 46 & 50 & 679 \\
\hline $\mathrm{N}^{0}$ OE CASES CHARGETO COURT & 72 & 81 & 93 & 70 & 84 & 87 & 81 & 64 & 66 & 241 & 67 & 72 & 978 \\
\hline
\end{tabular}

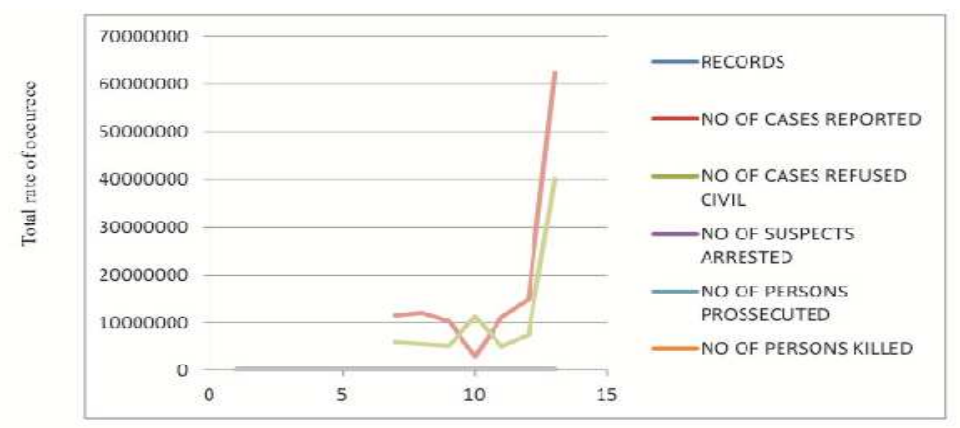

Figure 1(b): Frequency of Occurrence 
Table 3: Major Crimes and Local Acts Returns for the Edo State Police Command Headquarter 2011(January-December)

\begin{tabular}{|c|c|c|c|c|c|c|c|c|c|c|c|c|c|}
\hline MONTHS & IAN & FEB & MAR & $\mathrm{AP}$ & MAY & IUNE & JULY & $\mathrm{AU}$ & SEP & $O C T$ & $\mathrm{NOV}$ & $\mathrm{DEC}$ & TOTAL \\
\hline \multicolumn{14}{|l|}{ OFFEXCES } \\
\hline$\overline{\text { MURDER }}$ & 7 & 4 & 7 & 10 & 5 & 8 & 3 & 4 & 4 & 6 & 9 & 8 & 75 \\
\hline ARMROBBERY & 6 & 6 & 7 & 8 & 8 & 9 & 8 & 9 & 5 & 6 & 8 & 8 & 88 \\
\hline ATTEMPTMURDER & 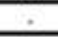 & - & 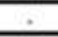 & 1 & 2 & 1 & 2 & . & 3 & $\therefore$ & $\therefore$ & 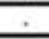 & 9 \\
\hline GRAVIOUS HARM WOUNDING & 14 & 15 & 12 & 13 & 13 & 14 & 16 & 16 & 12 & 14 & 16 & 13 & 168 \\
\hline ASSAULT & 22 & 23 & 24 & 23 & 22 & 21 & 23 & 24 & 23 & 21 & 24 & 19 & 269 \\
\hline \multicolumn{14}{|l|}{ STEALING } \\
\hline RAPEAND INDECENTG ASSAULT & 3 & 4 & 5 & 5 & 7 & 4 & 7 & 5 & 6 & 5 & 4 & 3 & 58 \\
\hline KIDNAPPING & 6 & 10 & 11 & 9 & 10 & 9 & 11 & 6 & 5 & 5 & 7 & 9 & 100 \\
\hline \multicolumn{14}{|l|}{ CULTISM } \\
\hline BURGLARY & 4 & 6 & 7 & 4 & 7 & 7 & 6 & 8 & 6 & 7 & 4 & 5 & 71 \\
\hline HOLSE BRAKING & 7 & 5 & 5 & 8 & 5 & 3 & 6 & 4 & 5 & 6 & 4 & 4 & 62 \\
\hline STORE BREAKING & - & 5 & 2 & 5 & 6 & 3 & $\therefore$ & 6 & 4 & 10 & 5 & 2 & 48 \\
\hline EALSE PRETENSE CHEATING & 11 & 13 & 12 & 10 & 11 & 14 & 12 & 10 & 8 & 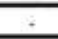 & 12 & 10 & 123 \\
\hline EORGERY & 2 & + & + & 1 & + & 1 & 1 & 1 & - & 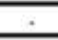 & - & $\therefore$ & 6 \\
\hline RECEIVING STOLEN PROPERTY & - & 1 & 1 & . & . & 1 & - & . & 1 & . & 3 & 1 & 8 \\
\hline UNLAWFUL POSSESSION & 1 & 1 & - & 2 & 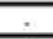 & 2 & 2 & . & 1 & 2 & 5 & 1 & 17 \\
\hline ARSON & 1 & + & 3 & . & 1 & 2 & - & 1 & - & 2 & $\therefore$ & - & 10 \\
\hline BREACH OF PUBLIC PEACE & 9 & 8 & 8 & 3 & 8 & 8 & 5 & 8 & 8 & 8 & 8 & 8 & 91 \\
\hline OFFENCES AGAINST FIREARMS & - & . & 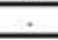 & 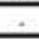 & - & $\therefore$ & - & . & - & - & - & + & . \\
\hline SXATCHED OF VEHICLES & + & $\therefore$ & - &. & - & - & $\therefore$ & $\cdot$ & - & + & $\therefore$ & $\therefore$ & + \\
\hline CAR THEFT & 26 & 21 & 26 & 20 & 25 & 24 & 26 & 20 & 20 & 24 & 37 & 22 & 291 \\
\hline VANDALIZATION & - & 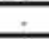 & - & . & 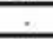 & $\therefore$ & 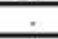 & 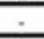 & $\therefore$ & . & - & 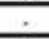 & $\therefore$ \\
\hline OTHER OFFENCES & 8 & 6 & 8 & 9 & 11 & 10 & 9 & 6 & 4 & 12 & 8 & II & 104 \\
\hline S.U.D & - & - & - & + & - & 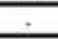 & - & . & - & - & - & $\therefore$ & - \\
\hline OFFENCES AGAINST TRAFFICACT & $\therefore$ & $\therefore$ & - & + & - & - & + & . & - & + & - & - & + \\
\hline MALICIOUSDAMAGE & 2 & $\dot{.}$ & - & . & 1 & - & 2 & . & 1 & . & - & 2 & 8 \\
\hline \multicolumn{14}{|l|}{ THREATENING VIOLENCE } \\
\hline COMMUNAL CLASH & $\therefore$ & - & - & . & + & - & + & . & - & 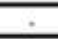 & 1 & + & 1 \\
\hline BOLTICAL VIOLENCE & $\therefore$ & $=$ & + & . & $\therefore$ & 1 & $\therefore$ & $\therefore$ & - & 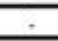 & $\therefore$ & $\therefore$ & 1 \\
\hline SUICIDE & 1 & - & 1 & 1 & 2 & 1 & - & - & - & 2 & - & - & 8 \\
\hline
\end{tabular}

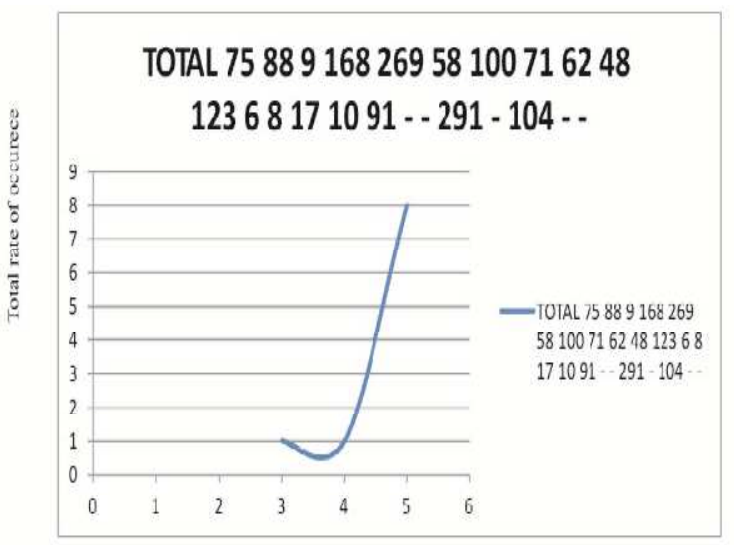

Figure 2(a): Frequency of Occurrence 
Table 4: Major Crimes and Local Acts Returns for the Edo State Police Command Headquarter 2011(January-December)

\begin{tabular}{|c|c|c|c|c|c|c|c|c|c|c|c|c|c|}
\hline MONTHS & JAN & FEB & MAR & $A P$ & MAY & JUNE & JUL & $\mathrm{AU}$ & SEP & OCT & $\mathrm{NOV}$ & $\mathrm{DEC}$ & TOTAL \\
\hline \multicolumn{14}{|l|}{ RECORDS } \\
\hline$N^{0}$ OF CASES REPORTED & 120 & 128 & 139 & 134 & 144 & 142 & 139 & 128 & 116 & 122 & 154 & 126 & 1592 \\
\hline \multicolumn{14}{|l|}{$\Lambda^{0}$ OF CASES REELISED CIVIL } \\
\hline $\mathrm{N}^{0}$ OE SUISPECTS ARRESTED & 136 & 131 & 130 & 146 & 121 & 123 & 149 & 112 & 115 & 142 & 157 & 135 & 1597 \\
\hline $\mathrm{I}^{0}$ OE PERSONS PROSSECUTED & 116 & 85 & 103 & 100 & 96 & 103 & 110 & 90 & 74 & 94 & 108 & 86 & 1165 \\
\hline$N^{0}$ OE PERSONS KILLED & 10 & 5 & 9 & 11 & 8 & 9 & 4 & 8 & 8 & 10 & 10 & 8 & 104 \\
\hline \multicolumn{14}{|l|}{$\perp^{0}$ OE PERSONS INIIIRED } \\
\hline VALUE OF PROPERTY STOLEN OR & 14868450 & 9978890 & 14331450 & 10803300 & 14854490 & 12504750 & 12827000 & 13785852 & 17025900 & 12618080 & 17856750 & 16060750 & 167512652 \\
\hline VALUE OF PROPERTY & 7421500 & 5272500 & 6450000 & 4084000 & 6835350 & 6384500 & 6170500 & 75788000 & 8483620 & 6235400 & 7455400 & 3650000 & 144230770 \\
\hline $\mathrm{N}^{0}$ OECASES UINDER & 42 & 43 & 53 & 44 & 61 & 46 & 44 & 61 & 50 & 46 & 76 & 60 & 616 \\
\hline$N^{0}$ OE CASES CHARGETO COLIRT & 88 & 72 & 86 & 85 & 83 & 96 & 95 & 67 & 66 & 76 & 78 & 66 & 958 \\
\hline
\end{tabular}

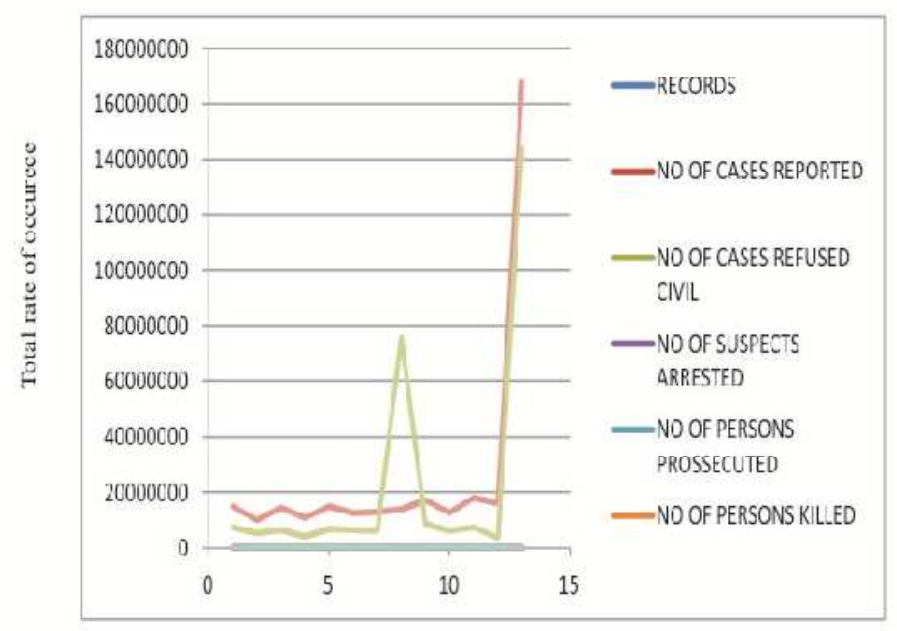

Figure 2(b): Frequency of Occurrence 
Table 5: Major Crimes and Local Acts Returns for the Edo State Police Command Head Quarter: 2012(January-December)

\begin{tabular}{|c|c|c|c|c|c|c|c|c|c|c|c|c|c|}
\hline MONTHS & JAN & FEB & MAR & $\mathrm{AP}$ & MAY & JUNE & JULY & $\mathrm{AU}$ & SEP & $\mathrm{OCT}$ & $\mathrm{NOV}$ & $\mathrm{DEC}$ & TOTAL \\
\hline \multicolumn{14}{|l|}{ OFFENCES } \\
\hline MURDER & 7 & 4 & 7 & 10 & 5 & 8 & 3 & 4 & 4 & 6 & 9 & 8 & 75 \\
\hline ARM/ROBBERY & 6 & 6 & 7 & 8 & 8 & 9 & 8 & 9 & 5 & 6 & 8 & 8 & 88 \\
\hline ATTEMPT/MURDER & - & - & $\therefore$ & 1 & 2 & 1 & 2 & - & 3 & - & - & - & 9 \\
\hline GRAVIOUS HARM/WOUNDING & 14 & 15 & 12 & 13 & 13 & 14 & 16 & 16 & 12 & 14 & 16 & 13 & 168 \\
\hline ASSAULT & 22 & 23 & 24 & 23 & 22 & 21 & 23 & 24 & 23 & 21 & 24 & 19 & 269 \\
\hline \multicolumn{14}{|l|}{ STEALING } \\
\hline RAPEAND INDECENTG ASSAULT & 3 & 4 & 5 & 5 & 7 & 4 & 7 & 5 & 6 & 5 & 4 & 3 & 58 \\
\hline KIDNAPPING & 6 & 10 & 11 & 9 & 10 & 9 & 11 & 6 & 5 & 5 & 7 & 9 & 100 \\
\hline \multicolumn{14}{|l|}{ CULTISM } \\
\hline BURGLARY & 4 & 6 & 7 & 4 & 7 & 7 & 6 & 8 & 6 & 7 & 4 & 5 & 71 \\
\hline HOUSE BRAKING & 7 & 5 & 5 & 8 & 5 & 3 & 6 & 4 & 5 & 6 & 4 & 4 & 62 \\
\hline STORE BREAKING & $\therefore$ & 5 & 2 & 5 & 6 & 3 & $\therefore$ & 6 & 4 & 10 & 5 & 2 & 48 \\
\hline FALSE PRETENSECCHEATING & 11 & 13 & 12 & 10 & 11 & 14 & 12 & 10 & 8 & - & 12 & 10 & 123 \\
\hline FORGERY & 2 & - & - & 1 & - & 1 & 1 & 1 & - & - & - & - & 6 \\
\hline RECEIVING STOLEN PROPERTY & - & 1 & 1 & - & - & 1 & - & - & 1 & - & 3 & 1 & 8 \\
\hline UNLAWFUL. POSSESSION & 1 & 1 & - & 2 & - & 2 & 2 & - & 1 & 2 & 5 & 1 & 17 \\
\hline ARSON & 1 & - & 3 & 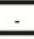 & 1 & 2 & - & 1 & - & 2 & $\therefore$ & $\therefore$ & 10 \\
\hline BREACH OF PUBLIC PEACE & 9 & 8 & 8 & 5 & 8 & 8 & 5 & 8 & 8 & 8 & 8 & 8 & 91 \\
\hline OFFENCES AGAINST FIRE ARMS & - & - & - & - & - & - & - & - & - & - & - & - & - \\
\hline SNATCHED OF VEHICLES & - & - & - & - & - & - & - & - & - & - & - & - & - \\
\hline CAR THEFT & 26 & 21 & 26 & 20 & 25 & 24 & 26 & 20 & 20 & 24 & 37 & 22 & 291 \\
\hline VANDALIZATION & - & - & - & - & - & - & - & - & - & - & - & - & - \\
\hline OTHER OFFENCES & 8 & 6 & 8 & 9 & 11 & 10 & 9 & 6 & 4 & 12 & 8 & 11 & 104 \\
\hline S.U.D & - & - & - & - & - & - & - & - & - & - & - & - & - \\
\hline OFFENCES AGAINST TRAFFIC ACT & - & - & - & - & - & - & - & - & - & - & - & - & - \\
\hline MALICIOUS DAMAGE & 2 & - & - & - & 1 & - & 2 & - & 1 & - & $\therefore$ & 2 & 8 \\
\hline \multicolumn{14}{|l|}{ THREATENING VIOLENCE } \\
\hline COMMUNAL CLASH & - & - & - & - & - & - & - & - & - & - & 1 & - & 1 \\
\hline POLITICAL VIOLENCE & - & - & - & - & - & 1 & - & - & - & - & - & - & 1 \\
\hline SUICIDE & 1 & - & 1 & 1 & 2 & 1 & - & - & - & 2 & - & - & 8 \\
\hline
\end{tabular}

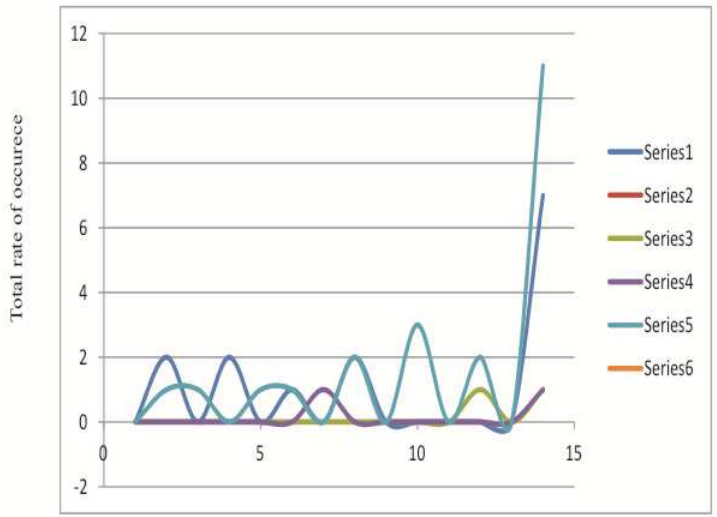

Figure 3(a): Frequency of Occurrence

Table 6: Major Crimes and Local Acts Returns for the Edo State Police Command Head Quarter: 2012(January-December)

\begin{tabular}{|c|c|c|c|c|c|c|c|c|c|c|c|c|c|}
\hline MONTHS & JAN & FEB & MAR & $\mathrm{AP}$ & MAY & JUNE & JUL & $\mathrm{AU}$ & SEP & OCT & NOV & $\mathrm{DEC}$ & TOTAL \\
\hline \multicolumn{14}{|l|}{ RECORDS } \\
\hline $\mathrm{N}^{0}$ OE CASES REPORTED & 84 & 122 & 121 & 115 & 113 & 126 & 102 & 137 & 114 & 115 & 140 & 110 & 1399 \\
\hline $\mathrm{N}^{0}$ OECASES REFUISED CIVIL & - & - & . & . & . & . & . & . & - & - & . & . & - \\
\hline $\mathrm{N}^{0}$ OE SUISPECTS ARRESTED & 71 & 129 & 116 & 108 & 124 & 123 & 63 & 115 & 103 & 99 & 138 & 94 & 1,283 \\
\hline$N^{0}$ OE PERSONS PROSSECUTED & 52 & 85 & 89 & 49 & 74 & 61 & 50 & 52 & 66 & 61 & 89 & 76 & 804 \\
\hline $\mathrm{N}^{0}$ OE PERSONS KILLED & 6 & 6 & 3 & 9 & 6 & 4 & 4 & 7 & 4 & 8 & 12 & 12 & 81 \\
\hline$N^{0}$ OE PERSONS INIURED & . & - & . & - & . & . & . & . & . & . & - & 1 & 1 \\
\hline VALUE OF PROPERTY STOLEN OR & $87,880,002,024$ & $11,041,350,68$ & $10,769,550,44$ & $12,040,500,38$ & 122837404 & $11,704,730,5$ & $11,114,050,03$ & 1392830048 & 124700005 & $140,050,004$ & 11124003 & $127,155,005$ & 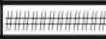 \\
\hline VALUE OF PROPERTY & 0 & 20,430 & 91700 & 87000 & 120300 & 180990 & 650000 & 81850 & 980000 & 987000 & 90000 & 410500 & $3,699,770$ \\
\hline $\mathrm{N}^{0}$ OE CASES INDER & 44 & 49 & 50 & 51 & 65 & 79 & 64 & 99 & 62 & 68 & 76 & 46 & 753 \\
\hline$N^{0}$ OE CASES CHARGE TO COLIRT & 40 & 69 & 71 & 64 & 58 & 47 & 38 & 38 & 52 & 47 & 64 & 64 & 652 \\
\hline
\end{tabular}




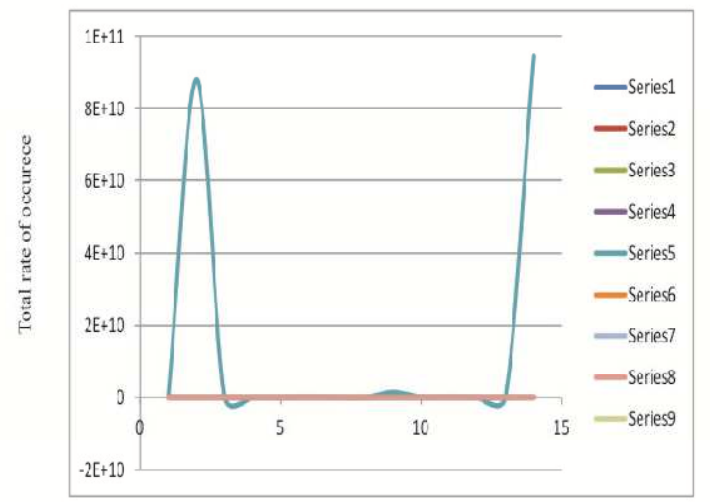

Figure 3(b): Frequency of Occurrence

Table 7: Major Crimes and Local Acts Returns for the Edo State Police Command Head Quarter: 2013(January-December)

\begin{tabular}{|c|c|c|c|c|c|c|c|c|c|c|c|c|c|}
\hline MONTHS & JAN & FEB & MAR & $\mathrm{AP}$ & MAY & JNE & JULY & $\mathrm{AU}$ & SEP & $\mathrm{OCT}$ & $\mathrm{NOV}$ & $\mathrm{DEC}$ & TOTAL \\
\hline \multicolumn{14}{|l|}{ OFFENCES } \\
\hline MURDER & 5 & 6 & 6 & 3 & 4 & 8 & 6 & 6 & 5 & 6 & 8 & 4 & 67 \\
\hline ARM:ROBBERY & 8 & 11 & 6 & 6 & 8 & 6 & 6 & 8 & 5 & 7 & 11 & 8 & 90 \\
\hline ATTEMPTMURDER & - & $\therefore$ & 4 & 1 & 3 & 2 & 3 & - & - & 3 & 2 & 1 & 19 \\
\hline GRAVIOUS HARM/WOUNDING & 17 & - & - & $\therefore$ & 9 & 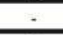 & $\therefore$ & 18 & 17 & 18 & 19 & 7 & 105 \\
\hline ASSAULT & 20 & 31 & 23 & 27 & 24 & 26 & 24 & 22 & 27 & 21 & 27 & 23 & 295 \\
\hline \multicolumn{14}{|l|}{ STEALING } \\
\hline RAPE AND INDECENTG ASSAULT & 5 & 4 & 5 & 5 & 5 & 11 & 12 & 10 & 11 & 7 & 22 & 3 & 100 \\
\hline KIDNAPPING & 3 & 9 & 11 & 5 & 7 & 3 & 7 & 11 & 9 & 5 & 7 & 3 & 80 \\
\hline \multicolumn{14}{|l|}{ CULTISM } \\
\hline BURGLARY & 5 & 6 & 4 & 4 & 6 & 4 & 9 & 3 & 11 & 8 & 7 & 8 & 71 \\
\hline HOUSE BRAKING & 4 & 4 & 3 & 5 & 6 & 9 & 7 & 4 & 7 & 5 & 8 & 6 & 68 \\
\hline STORE BREAKING & 7 & 3 & 5 & 3 & 4 & II & 9 & 5 & 6 & 7 & 7 & 8 & 73 \\
\hline FALSE PRETENSE/CHEATING & 12 & 8 & 7 & 6 & 10 & 8 & 13 & 12 & 10 & 15 & 12 & 9 & 122 \\
\hline FORGERY & - & 1 & 1 & - & - & 2 & 1 & 1 & 1 & - & $\dot{-}$ & $\dot{-}$ & \\
\hline RECEIVING STOLEN PROPERTY & $\therefore$ & - & - & - & 2 & 2 & 1 & 1 & 2 & 3 & - & 1 & 12 \\
\hline UNLAWFUL. POSSESSION & 5 & 7 & 4 & 3 & 4 & 6 & 5 & 4 & 7 & 4 & 4 & 3 & 56 \\
\hline ARSON & 2 & 1 & - & 2 & - & 1 & 2 & - & . & . & 2 & 1 & 11 \\
\hline BREACH OF PUBLIC PEACE & 8 & 7 & 5 & 4 & 7 & 6 & 8 & 5 & 8 & 6 & 8 & 5 & 77 \\
\hline \multicolumn{14}{|l|}{ OFFENCES AGAINST FIRE ARMS } \\
\hline \multicolumn{14}{|l|}{ SNATCHED OF VEHICLES } \\
\hline CAR THEFT & 23 & 21 & 21 & 15 & 21 & 24 & 21 & 21 & 23 & 24 & 24 & 23 & 261 \\
\hline \multicolumn{14}{|l|}{ VANDALIZATION } \\
\hline \multicolumn{14}{|l|}{ OTHER OFFENCES } \\
\hline \multicolumn{14}{|c|}{ S.U.D } \\
\hline \multicolumn{14}{|l|}{ OFFENCES AGAINST TRAFFIC ACT } \\
\hline MALICIOUS DAMAGE & $\therefore$ & 6 & 6 & 6 & 14 & 14 & 18 & 15 & 13 & 15 & 17 & 11 & 145 \\
\hline THRFATENING VIOI.FNCF & - & 7 & 5 & 5 & 12 & 20 & 14 & 17 & 15 & 12 & 14 & 10 & 141 \\
\hline \multicolumn{14}{|l|}{ COMMUNAL CLASH } \\
\hline POLITICAL VIOLENCE & & & & & & & & & & & & & \\
\hline
\end{tabular}

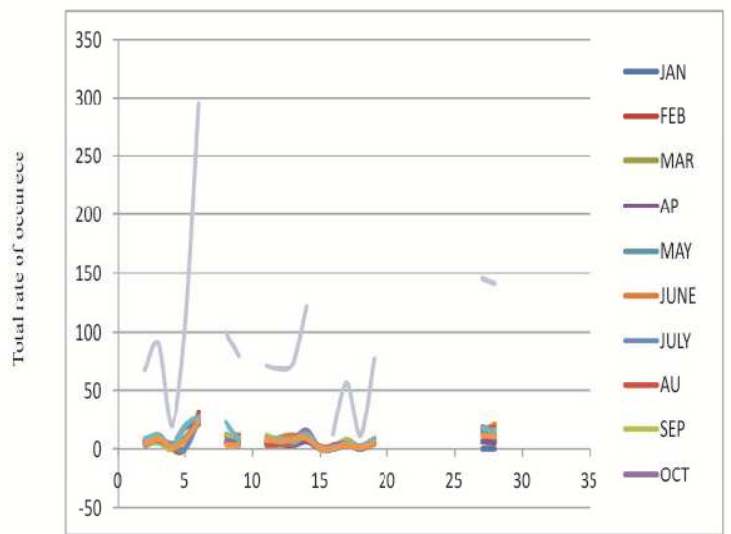

Figure 4(a): Frequency of Occurrence 
Table 8: Major Crimes and Local Acts Returns for the Edo State Police Command Head Quarter: 2013(January-December)

\begin{tabular}{|c|c|c|c|c|c|c|c|c|c|c|c|c|c|}
\hline MONTHS & JAN & FEB & MAR & $\mathrm{AP}$ & MAY & JUNE & JUL & $\mathrm{AU}$ & SEP & $\mathrm{OCT}$ & $\mathrm{NOV}$ & DEC & TOTAL \\
\hline \multicolumn{14}{|l|}{ RECORDS } \\
\hline $\mathrm{N}^{0}$ OF CASES REPORTED & 126 & 132 & 116 & 100 & 146 & 172 & 176 & 162 & 177 & 166 & 200 & 133 & 1806 \\
\hline $\mathrm{N}^{0}$ OE CASES REFUSED CIVI & - & - & - & - & - & - & - & 1 & - & - & 5 & 2 & 8 \\
\hline $\mathrm{N}^{0} \mathrm{~N}$ E SISPECTS ARRESTED & 128 & & & & & & & & & & & & \\
\hline $\mathrm{S}^{0}$ OF PERSONS PROSSECUTED & 68 & & & & & & & & & & & & \\
\hline $\mathrm{N}^{0} \mathrm{O}$ PERSONS KILLED & 7 & & & & & & & & & & & & \\
\hline \multicolumn{14}{|l|}{ No OE PERSONS INIURED } \\
\hline VALUE OF PROPERTY STOLEN OR & $1931450)$ & & & & & & & & & & & & \\
\hline VALUE OF PROPERTY & 6834000 & & & & & & & & & & & & \\
\hline N OF CASES INDER & 80 & 75 & 74 & 60 & 83 & 94 & 82 & 84 & 84 & 82 & 84 & 78 & 960 \\
\hline $\mathrm{YO}^{0} \mathrm{OF}$ CASES CHARGEDTO & 46 & 57 & 42 & 40 & 63 & 78 & 94 & 77 & 93 & 84 & 111 & $5 \hat{3}$ & 838 \\
\hline
\end{tabular}

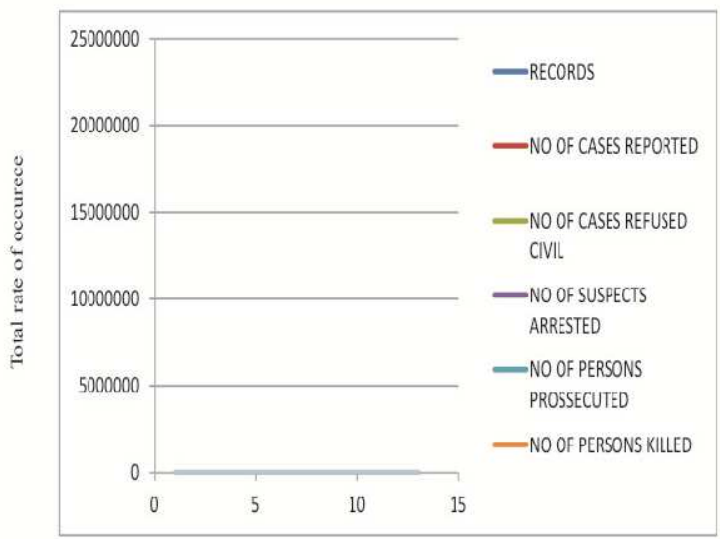

Figure 4(b): Frequency of Occurrence

Table 9: Major Crimes and Local Acts Returns for the Edo State Police Command Head Quarter: 2014(January-December)

\begin{tabular}{|c|c|c|c|c|c|c|c|c|c|c|c|c|c|}
\hline MONTHS & JAN & FEB & MAR & $\mathrm{AP}$ & MAY & JUNE & JULY & $\mathrm{AU}$ & SEP & DCT & $\mathrm{NOV}$ & $\mathrm{DEC}$ & TOTAL \\
\hline \multicolumn{14}{|l|}{ OFFENCES } \\
\hline MURDER & 10 & 7 & 10 & 19 & 11 & 6 & 5 & 4 & 3 & 8 & 4 & 5 & 92 \\
\hline ARMROBRERY & 4 & 5 & 5 & 6 & 5 & 3 & 4 & 5 & 2 & 8 & 4 & 5 & 60 \\
\hline ATTEMPT/MURDER & 1 & 1 & 1 & . & 4 & 1 & 2 & - & - & 2 & 2 & 1 & 15 \\
\hline GRAVIOUS HARMWOUNDING & 16 & 14 & 18 & 15 & 127 & 28 & 42 & 50 & 45 & 34 & 23 & 9 & 412 \\
\hline ASSAULT & 24 & 2. & 23 & 27 & 141 & 47 & 52 & 56 & 32 & 18 & 37 & 17 & 478 \\
\hline STEALING & 28 & 24 & 27 & 30 & 163 & 34 & 42 & 52 & 46 & 38 & 26 & 14 & 510 \\
\hline RAPEAND INDECENTG ASSAULT & 14 & 14 & 7 & 10 & 30 & 4 & 7 & 11 & 13 & 3 & 4 & - & 121 \\
\hline KIDNAPPING & 5 & 8 & 3 & 2 & 6 & 2 & 2 & 2 & 2 & 3 & 2 & 2 & 39 \\
\hline \multicolumn{14}{|l|}{ CULTISM } \\
\hline BURGLARY & 7 & 7 & 6 & 8 & 9 & 7 & 9 & 7 & 5 & 3 & 15 & 7 & 90 \\
\hline HOUSE BRAKING & 5 & 6 & 4 & 6 & 23 & 5 & 7 & 8 & 4 & 2 & 4 & 1 & 75 \\
\hline STORE BREAKING & 3 & 5 & 6 & 5 & 13 & 4 & 6 & 7 & 5 & 1 & 5 & 2 & 62 \\
\hline FALSE PRETENSECCHEATING & 17 & 12 & 10 & 11 & - & 13 & 9 & 12 & 8 & 5 & 3 & 1 & 101 \\
\hline FORGERY & - & . & 1 & $\cdot$ & . & - & 1 & . & - & 1 & . & - & 3 \\
\hline RECEIVING STOLEN PROPERTY & 3 & 1 & 1 & 1 & 1 & - & - & 2 & - & - & 3 & - & 12 \\
\hline UNLAWFUL. POSSESSION & 3 & 2 & 4 & 3 & 7 & 2 & 2 & 4 & - & - & 1 & 2 & 30 \\
\hline ARSCN & 1 & 3 & 3 & 2 & - & - & 2 & - & 1 & 1 & - & 1 & 14 \\
\hline BREACH OF PUBLIC PEACE & 6 & 7 & 5 & 19 & 70 & 21 & 25 & 32 & 18 & 22 & 14 & 9 & 248 \\
\hline OFFEVCES AGAINST FIRE ARMS & - & - & - & . & $\dot{.}$ & - & - & - & - & - & - & - & - \\
\hline SNATCHED OF VEHICLES & - & - & - & . & - & - & - & - & - & - & - & - & - \\
\hline CAR THEFT & . & - & . & . & - & - & - & - & - & - & - & - & - \\
\hline VANDALIZATION & - & - & - & - & - & - & - & - & - & - & - & - & - \\
\hline OTHER OFFENCES & - & - & - & - & - & - & - & - & - & - & - & - & - \\
\hline S.U.D & - & - & - & - & - & - & - & - & - & - & - & - & - \\
\hline OFFENCES AGAINST TRAFFICACT & - & - & - & - & - & - & - & - & - & - & - & - & - \\
\hline MALICIOUS DAMAGE & 21 & 15 & 18 & 22 & 59 & 9 & 14 & 18 & 9 & 6 & 9 & 2 & 202 \\
\hline THREATENING VIOLENCE & 14 & 16 & 14 & 11 & 24 & 14 & 17 & 21 & 16 & 20 & 17 & 11 & 195 \\
\hline COMMUNAL CLASH & - & - & - & - & - & - & - & - & - & - & 1 & - & 1 \\
\hline POLITICAL VIOLENCE & - & - & - & - & - & 1 & - & . & - & $\dot{-}$ & . & - & 1 \\
\hline
\end{tabular}




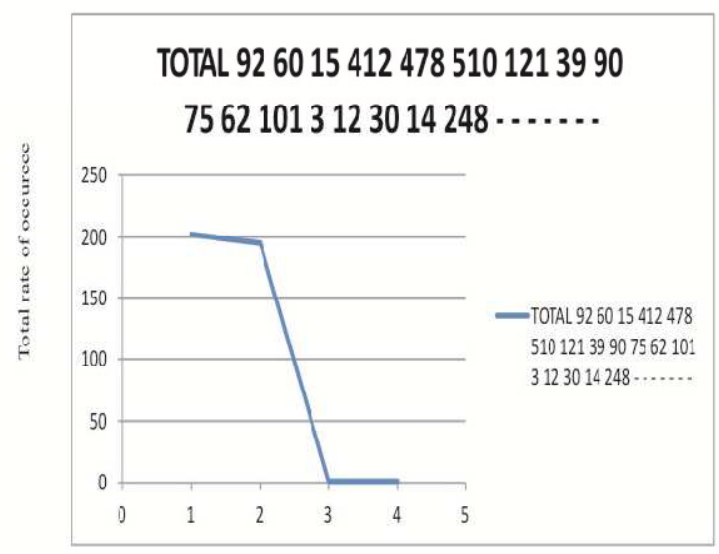

Figure 5(a): Frequency of Occurrence

Table 10: Major Crimes and Local Acts Returns for the Edo State Police Command Head Quarter: 2010(January-December)

\begin{tabular}{|c|c|c|c|c|c|c|c|c|c|c|c|c|c|}
\hline MONTHS & JAN & FEB & MAR & $A P$ & $\mathrm{MAY}$ & JUNE & 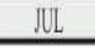 & $\mathrm{AU}$ & SEP & $\mathrm{OCT}$ & $\mathrm{NOV}$ & DEC & TOTAL \\
\hline \multicolumn{14}{|l|}{ RECORDS } \\
\hline NOECASES REPIRTED) & 182 & 168 & 166 & 197 & 693 & $\$ 15$ & 248 & 291 & 208 & 177 & 174 & 92 & 2911 \\
\hline LOAfCASES REFISEDCTIII & 6 & 3 & 2 & 1 & 39 & 22 & 41 & 31 & 24 & 14 & 22 & 15 & 226 \\
\hline Norsuspects ararested) & 1.52 & 144 & 100 & 136 & 496 & 2114 & 187 & $14 i$ & 169 & 142 & 156 & 102 & 2133 \\
\hline noepersons prossecuted & 112 & 104 & 82 & $9 \%$ & 401 & 43 & 135 & 112 & 103 & 58 & $7 ?$ & 68 & 1486 \\
\hline LOAF PERSONS KIILLED & 10 & 7 & 10 & 19 & 11 & 6 & 5 & 4 & 3 & 14 & 4 & 5 & 48 \\
\hline AOE PERSONSINULRED & 16 & 14 & 18 & 15 & 128 & 28 & 45 & 50 & 4.5 & 37 & 23 & 9 & 428 \\
\hline VALUE OF PROPERTY STOLENOR & $11,836,940$ & $18,618,700$ & $43,026,00$ & $51,080,790$ & $215,169,720$ & $98,509,450$ & $9: 236,400$ & $128,220,650$ & $2,900,190$ & $19,348,260$ & $46,136,120$ & $16,045,980$ & $879,661,660$ \\
\hline VALUE OP PROPERTY & $0,156,0000$ & $13,060,000$ & $15,828,000$ & $20,040,790$ & $111,000,000$ & $412,326,000$ & $42,522.300$ & $83,046,000$ & 19,100,000 & $4,550,100$ & 12.789.140 & 5,862520 & $383,487,450$ \\
\hline Nofcasesinder & 72 & 76 & 93 & 114 & 331 & 83 & 87 & 157 & 115 & 120 & 103 & 48 & 1280 \\
\hline No oecaseschargeto colirt & 104 & 89 & 71 & $7 j$ & 323 & 94 & 109 & 107 & 70 & 44 & 49 & 29 & 1273 \\
\hline
\end{tabular}

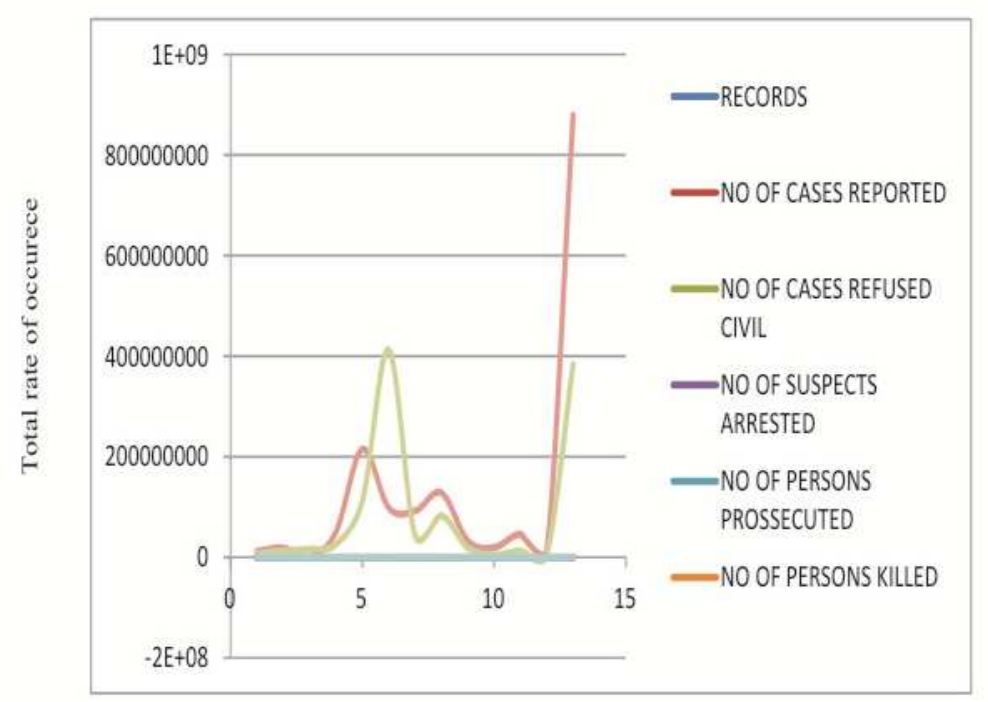

Figure 5(b): Frequency of Occurrence 
Table 11: Major Crimes and Local Acts Returns for the Edo State Police Command Head Quarter: 2015(January-December)

\begin{tabular}{|c|c|c|c|c|c|c|c|c|c|c|c|c|c|}
\hline MONTHS & JAN & FEB & MAR & $\mathrm{AP}$ & $\mathrm{MAY}$ & JUNE & $\mathrm{JUL}$ & $\mathrm{AL}$ & SEP & $\mathrm{OCT}$ & $\mathrm{NOV}$ & $\mathrm{DEC}$ & TOTAL \\
\hline OFFENCES & & & & & & & & & & & & & \\
\hline MURDER & 6 & 17 & 11 & 6 & 8 & 3 & 3 & 5 & 4 & 4 & 4 & 1 & 77 \\
\hline ARM/ROBBERY & 10 & 5 & 6 & 4 & 11 & 3 & 4 & 5 & 7 & 3 & 1 & 1 & 65 \\
\hline ATTEMPT/MURDER & 1 & 2 & 3 & 2 & 6 & 6 & 2 & 2 & 2 & 1 & - & - & 27 \\
\hline GRAVIOUS HARM/WOUNDING & 3 & 8 & 12 & 16 & 23 & 38 & 23 & 19 & 16 & 4 & 11 & 14 & 187 \\
\hline ASSAULT & 18 & 26 & 19 & 24 & 3 & 94 & 22 & 27 & 21 & 20 & 23 & 17 & 316 \\
\hline SIEALING & 22 & 38 & 17 & 23 & 31 & 26 & 22 & 18 & 13 & 28 & 22 & 19 & 248 \\
\hline RAPEAND INDECENTG ASSAULT & 6 & 4 & 8 & 3 & 4 & 6 & 1 & 2 & 2 & 5 & 4 & 1 & 45 \\
\hline KIINAPPING & 2 & 4 & 4 & 2 & 5 & 4 & 1 & 4 & 2 & 4 & 3 & 1 & 36 \\
\hline CULTISM & - & - & - & - & - & - & 5 & 7 & 18 & 5 & 1 & - & 36 \\
\hline BURGRARY & 3 & 7 & 5 & 8 & 6 & 4 & 2 & 1 & 2 & 4 & 1 & . & 43 \\
\hline HOUSE BRAKING & 2 & 5 & 7 & 5 & 3 & 1 & 4 & . & $\therefore$ & 5 & 3 & - & 35 \\
\hline S/BREAKING & 3 & 4 & 7 & 4 & $i$ & 2 & 2 & . & 2 & 6 & 5 & - & 42 \\
\hline FALSE PRETENSE/CHEATING & 20 & 18 & 17 & 9 & 13 & 9 & 12 & 6 & 9 & 3 & 7 & 5 & 128 \\
\hline FORGERY & 3 & . & 1 & - & 1 & - & - & . & $\cdot$ & 1 & - & - & 6 \\
\hline RECFIVING STOL.EN PROPERTY & . & 2 & - & - & 2 & 4 & - & - & - & 2 & 3 & 1 & 14 \\
\hline UNL. POSSESSION & $?$ & 4 & 1 & 4 & 7 & 9 & 4 & 6 & 8 & 3 & - & - & 53 \\
\hline ARSDN & 2 & 1 & . & 2 & . & . & 1 & - & - & . & 1 & - & 7 \\
\hline BREACH OH PUBLIC PEACE & 8 & 15 & 5 & 9 & 13 & 11 & 9 & 5 & 15 & 13 & 6 & 8 & 17 \\
\hline OFFENCES AGAINST FIRE ARMS & $\therefore$ & - & 6 & - & $\therefore$ & - & 3 & - & 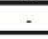 & . & - & - & 9 \\
\hline SNATCHFD OF VFHICIFS & . & - & - & - & . & - & - & - & - & . & - & - & - \\
\hline CAR THEFT & . & - & - & - & - & - & - & - & - & - & - & - & - \\
\hline VANDALIZATION & . & - & - & - & - & - & - & - & - & - & - & - & - \\
\hline OTHER OFFENCES & - & - & - & - & - & - & 16 & 2 & - & - & 9 & 6 & 40 \\
\hline S.U.D & . & 4 & - & - & - & - & - & - & - & - & - & - & 4 \\
\hline OFFENCES AGAINST TRAFFIC ACT & . & - & 1 & - & - & - & - & - & - & 7 & - & - & 1 \\
\hline
\end{tabular}

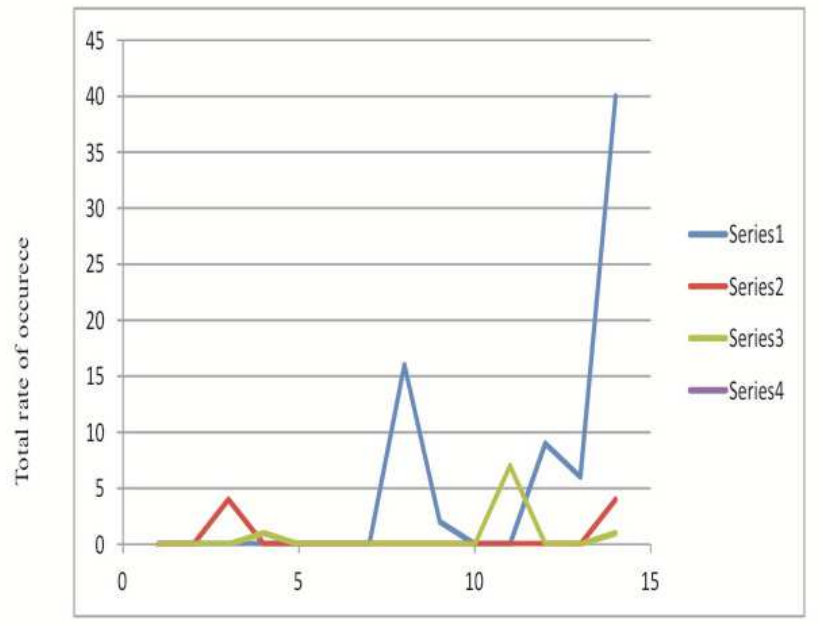

Figure 6(a): Frequency of Occurrence

Table 12: Major Crimes and Local Acts Returns for the Edo State Police Command Head Quarter: 2015(January-December)

\begin{tabular}{|c|c|c|c|c|c|c|c|c|c|c|c|c|c|}
\hline MONTHS & JAN & FEB & MAR & $A P$ & MAY & JUNE & UL & $\mathrm{AU}$ & SEP & OCT & $\mathrm{NOV}$ & $\mathrm{DEC}$ & TOTAL \\
\hline \multicolumn{14}{|l|}{ RECORDS } \\
\hline $\mathrm{N}^{0}$ OECASES REPORTED & 118 & 192 & 115 & 121 & 175 & 281 & 201 & 100 & 120 & 120 & 105 & 74 & 1733 \\
\hline $\mathrm{N}^{0}$ OECASES REEERED CIVIL. & 22 & 29 & 27 & 15 & 36 & $5 ?$ & 46 & 25 & 33 & 29 & 23 & 9 & 347 \\
\hline$\sum^{0}$ OE PERSONS ARRESTED & 70 & 50 & 168 & 98 & 114 & 153 & 105 & 114 & 46 & 81 & 39 & 45 & 1089 \\
\hline $\mathrm{X}^{0}$ OL PERSONS PROSSECLITLD & 24 & 50 & 101 & 62 & 74 & 76 & 42 & 40 & 36 & 47 & 28 & 21 & 501 \\
\hline $\mathrm{N}^{0}$ OH PEHSONS KILLHI) & 6 & 19 & 14 & 6 & 8 & 10 & 3 & 5 & 4 & 4 & 9 & 1 & 89 \\
\hline $\mathrm{N}^{0}$ OE PERSONS INUIRED & 4 & 8 & 14 & 23 & 27 & . & 30 & 24 & 21 & 20 & 14 & 14 & 199 \\
\hline VALUE OF PROPERTY STOLEN & 14677000 & 271296250 & 15877575 & 23339157 & 534188050 & 10597340 & 93442350 & 8233000 & 4676450 & 10905345 & $43,140,000$ & . & $1,130,374,517$ \\
\hline VALUE OF PROPERTY & 650,000 & $\therefore$ & $\therefore$ & . & $\therefore$ & $\therefore$ & 91.000 & $\therefore$ & $\therefore$ & $\therefore$ & $4,000,000$ & $\therefore$ & 4091000 \\
\hline$N^{0}$ OFCASES INDER & 73 & 111 & 74 & 83 & 78 & 185 & 136 & 52 & 54 & 63 & 58 & 45 & 967 \\
\hline$N^{0}$ OF Cases Charge TO COURT & 23 & 52 & 24 & 23 & 61 & 44 & 19 & 23 & 33 & 28 & 24 & 16 & 354 \\
\hline
\end{tabular}




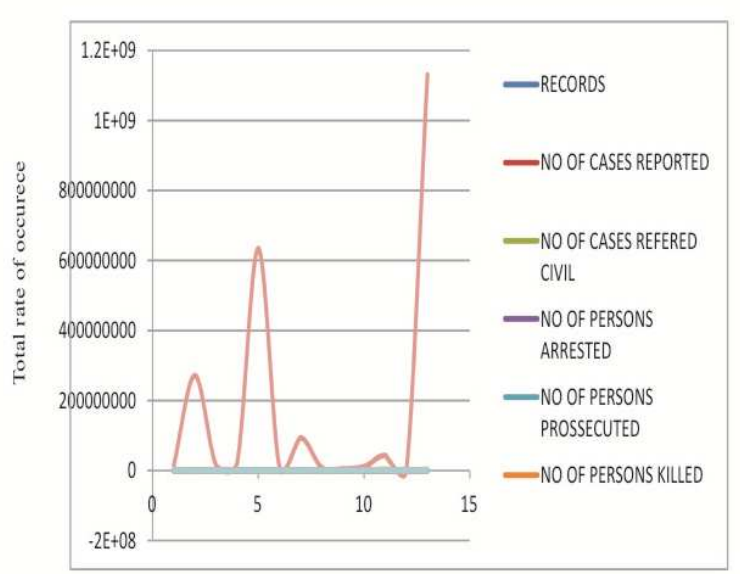

Figure 6(b): Frequency of Occurrence

\section{DISCUSSIONS OF RESULTS}

The following shows the analysis of crime rate, as it occurs over a period of six years (2010 to 2015). In the Edo State police headquarters inside state CID.

Tables 1 to 12 above shows the number of offenses recorded in from 2010 to 2015 ranging from Murder to Suicide.

\section{Offense Related With Stealing}

The figures above $2 \mathrm{a}, 3 \mathrm{a}$ and $4 \mathrm{a}$ showed that there was no case of stealing in the years 211,212 and 2013; it was less significant in the year 2010 figure 1a, significant in the year 2015 figure 6a, and dominant in the year 2014 figure 5a. It may also be observed in the case of burglary that it occurred at a constant rate all through the years 2010,2011,2012,2013 and 2015 figures 1a, 2a, 3a, 4a, and 6a; it was a bit dominant in the year 2014 figure 5a. The case of house braking occurred in the approximated rate in the years 2011, 2012 and 2015, figures 2a, 3a and 6a dominant in the years 2010, 2014, and 2015 figures 1a, 5a and 6a. The case of store breaking occurred at a constant rate through the years 2010 to 2012, 2015 figures 1a, 3a, and 6a. It was dominant in the years 2013 and 2014 figures 4a and 5a. Car theft did not occur in the years 2014 and 2015figures 5a and 6a but was dominant through the years 2010 to 2013 ranging from 200 to 250 figures 1a and 3a. An arm / robbery occurs almost at a constant rate throughout the years 2010, 2014 and 2015 figures 1a,5a and 6a, appear dominant through the years 2011 to 2013 figures $2 \mathrm{a}$ and $4 \mathrm{a}$.

\section{Offense Related With Murder}

Figures 4a and 6a, above showed that there was the case of suicide was insignificant in the years 2013 to 2015 , significant in the year 2012 figure 3a. Attempt/murder was less significant in the years 2012 and 2014 figures 3a and 5a, significant in the years 2010, 2014 figures 1a and 5a; it was dominant in the years 2013 and 2015 figures 4a and 6a. A murder occurred significantly all through the years 2010 to 2015 figures 1a and 6a; the frequency of occurrence is approximately (75) in 2010, 2013; (105) in 2011, 2012 and 2015, but dominant (145) in 2014. Kidnapping is also significant all through the years 2010 to 2015, but the frequency of occurrence varies approximately from (50) in the years 2014, 2015; (75) in the years 2010 and 2013 (105) in the year 2011 to (130) in the year 2012. 


\section{Offense Related With Violence}

Figures 2a and 3a above showed that threatening of violence did not occur in the years 2011, 2012; it was insignificant in the year 2010 signed in the year 2014 and dominant in the year 2015. Political violence did not occur in the years 2013, 2015; then it was insignificant with the frequency of occurrence of about (20) through the years 2010, 2011, 2012 and 2014. Communal clash did not occur in the years 2013, 2015; then it was insignificant with the frequency of occurrence of about (20) through the years 2010, 2011, 2012 and 2014. Vandalization did not occur all through the years 2011 to 2015 and it appears significantly in the year 2010. Malicious damage did not occur in the years 2015; appear insignificant in the years 2010, 2011, 2012, signed in the year 2013 and dominant in the year 2014.

\section{Offense related with Societal Instability}

Figures 1a to 6a above showed that cultism did not occur all through the years 2011 to 2014, but it was insignificant in the year 2010 and a bit dominant in the year 2015. Offenses against the firearm act did not occur all through the years 2011 to 2014, but it was insignificant in the year 2010 and a bit dominant in the year 2015. Rape and indecent assault occurred all through the years 2010 to 2015 with the frequency of occurrence varying approximately from (50) in the year 2015; (70) in the years 2011, 2012; (75) in the year 2015; (205) in the year 2013 to (300) in the year 2014. Breach of public peace occurred all through the years 2010 to 2015 with the frequency of occurrence varying approximately from (50) in the year 2015; (105) in the years 2012, 2013; (205) in the years 2010, 2011 to (400) in the year 2014. The assault occurred all through the years 2010 to 2015 with the frequency of occurrence varying approximately from (300) in the year 2012, (350) in the years 2011, 213, 2015; (450) in the year 2010 to (550) in the year 2014.

\section{Offense related with Societal Cheating}

Figures 2a to 6a, above shows that S.U.D did not occur all through the years 2011 to 2014, it was insignificant in the year 2010 and a little bit significant in the year 2015. An offense against traffic act did not occur all through the years 2011 to 2014; it was insignificant in the year 2010 and a little bit significant in the year 2015. The case of forgery did not occur in the year 2013; it was insignificant in the year 2014, a little bit significant in the year 2011, more significant in the year 2010, 2015 and a bit dominant in the year 2012. The case of receiving stolen property occurred all through the years (2010 to 2015) with the frequency of occurrence ranging approximately from (20) in the year 2010, (25) in the years 2011,2012; (50) in the years 2013, 2014 to (52) in the year 2015. Arson offenses occurred all through the years (2010 to 2015 ) with the frequency of occurrence ranging approximately from (25) in the year 2015, (35) in the year 2013, (40) in the years $2010,2011,2013$ to (45) in the year 2014. Unlawful possession offenses occurred all through the years (2010 to 2015) with the frequency of occurrence ranging approximately from (20) in the year 2011, (30) in the year 2012, (45) in the years 2010, 2014, (90) in the years 2013,2015. Other offenses did not occur in the years 2013, 2014; it was significant in the years 2012, 2015, dominant in the years 2010, 2011 with the frequency of occurrence of about 140. False pretense / cheating occurred dominantly all through the years with the frequency of occurrence ranging approximately from (100) in the year 2014, (105) in the year 2012, (1340) in the years 2011, 2013 to (148) in the years 2010 to 2015.

Figure $1 \mathrm{~b}$ to $6 \mathrm{~b}$ above showed the number of cases reported from 2010 to 2015 on yearly plot.

Figure $3 \mathrm{~b}$ and $6 \mathrm{~b}$ showed that the year 2012 and 2015 witness the lowest number of cases reported.

Figure 1b, 2b and 4b showed that there were 1500 to 2000 cases reported in the year 2010, 2011 and 2013. 
Figure 5b showed that 2014 recorded the highest cases reported under the period review.

Also, it would be observed as shown by the figures 1 to 6 that, there were no cases recorded in the area of snatching of vehicles and vandalization in the year 2010 to 2015 .

It was documented that there few cases of offenses recorded in the area of attempt to murder, cultism, forgery, receiving of stolen property, unlawful possession of arms, arson, offenses against fire arm, S.U.D, offenses against traffic act, communal clash, political violence and suicide in the period of 2010 to 2015.

However, there were several cases recorded by the security authorities in the area of murder, arm robbery, rape and indecent assault, kidnapping, burglary, housebreaking, store breaking, other offenses, malicious damage and threatening violence in the period of 2010 to 2015.

It would also be documented that there were increasing cases in the area of grievous harm, assault offenses, stealing, false pretense/cheating, breach of the public peace and car theft in the year, 2010 to 2015.

It should be suggested that the security agencies should persecute those involve in the area of grievous harm, assault offenses, stealing, false pretense/cheating and car theft in the society.

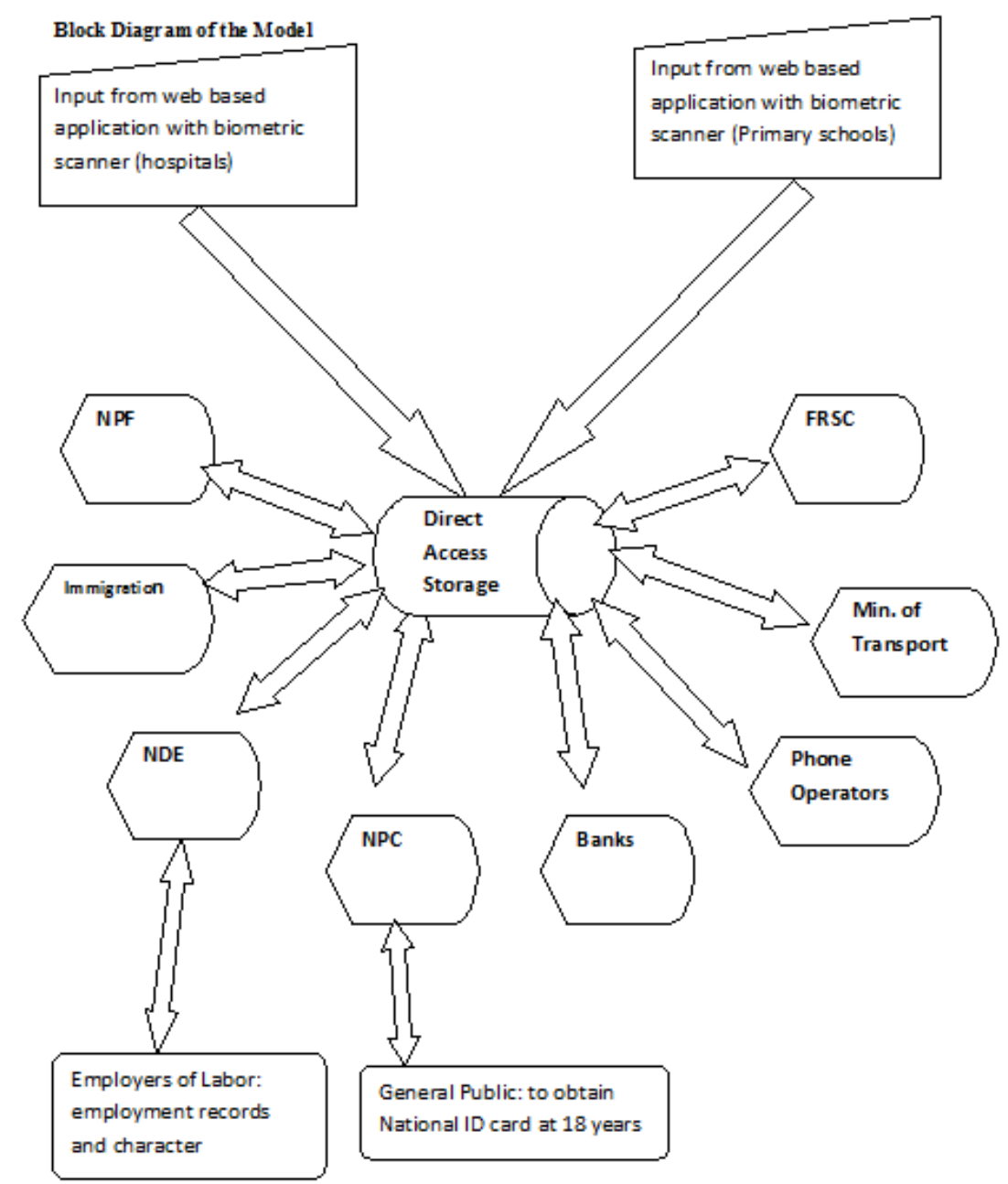

Figure 1.0: Block Diagram of the Direct Access Storage Model System

Source: field of authors 
For the purpose of the project, a domain may be registered to host the application and create other sub domains for the key sectors, to illustrate, how the data gathered will be sent across to other domains and used by the different sectors?

\section{CONCLUSIONS}

From the above analysis, it is found that even after prosecution, some of the bailed offenders continue with the same activities, without being properly traced. The security agents rely solely on the public willingness and information to track down the wanted offenders at large, as stated by the Edo State commissioner of police, Sir Foluso Adebanjo, during the Ebs TV program Crime Watch show I quote “Remember, the police need your information's to help you”. At the moment, such offense occurred if the data warehouse is being connected to the direct access storage, whether at large or not, all offenders' bioinformation, finger print, passport photograph etc. will be known, a situation that can make investigation easy.

\section{REFERENCES}

1. Biometrics for Nigeria retrieved from www.aware.com/Software Products - Fingerprint, Face, Iris. On the 2013-06-10.

2. Biometric www.m2sys.com/Biometric-SDK, Fingerprint, Finger/Palm Vein \& Iris; 1Server, No SDK. On the 2010-06-10.

3. "Biometrics (2007). Overview "retrieved from Biometrics.cse. msu. edu. on the 2012-06-10.

4. Bill Flook (2013). "This is the 'biometric war' Michael Saylor was talking about". Washington Business Journal; 3:56

5. "Biometrics for Secure Authentication". Retrieved from www.m2sys.com/Biometric-DK on the 2012-(2009).

6. "Characteristics of biometrics of biometric". Cernet.

7. Esselaar, P.;O. Hesselmark; T. James. and j. Miller (2001). Final report: A three-country ICT survey for Rwanda, Tanzania, and Mozambique. Retreived from http://www.trigrammic.com/downloads/SIDA\%20Final\%20Project\%20Report.pdf on the $21 / 7 / 2010$

8. Festus Alenkhe; (2016). A security Mirror of the Nigerian Society EBS Tv. Show "Crime Watch":14-22.

9. Gary P. B.;A. D. Keinath; P. Hernandez; L. Master; R. Thurston(2009). Element Distribution Modeling] "Biometrics(2007). Overview" A Primer; (Version 2), Natureserve, Arlington, Virginia, 62-68.

10. Hand, D.; H. Mannila; P. Smyth(2001). Principles of Data Mining. MIT Press, Cambridge MA, pp. 5-25.

11. Jain, A.; L.Hong and S. Pankanti (2000). "Biometric Identification". Communications of the ACM. 43(2): 91-98

12. Jain, A.K.; R. Bolle and S. Pankanti, S. (1999). Biometrics: Personal Identification in

13. Networked Society. Kluwer Academic Publications; 2: 95-98 